\title{
The Optimal Control Based on Particle Swarm Intelligence Algorithm
}

\author{
Yun Zhou \\ School of North China Electric Power University, Baoding 071000, China; \\ 15028260178@163.com
}

Keywords: PSO algorithm, Intelligent control

\begin{abstract}
In order to further investigate the intelligent optimization of particle swarm optimization (pso) algorithm, we carried on the design experiments in this paper, the particle swarm optimization (pso) algorithm, the position of the first set search point X0i and speed V0i initialization;Then evaluate each particle, calculate the fitness value of particles, if is better than that of the particle current individual extremum, update the individual extremum, if in the particles of all particles in the neighborhood of the best good of individual extremum in the current record the serial number of the particles, and the update function value;For each particle velocity and position updating;The end of the final inspection is in line with the conditions, if the current number of iterations to achieve the pre-set number of maximum (or minimum error requirement), is to stop the iteration, output the optimal solution, or go to the evaluation steps, particles will eventually take the simulation control object, carries on the simulation, intelligent optimization, particle swarm algorithm is verified in engineering has a great application prospect.
\end{abstract}

\section{Background}

Particle swarm optimization (PSO) algorithm is by Kennedy and Eberhart in 1995 with a simple computer simulation of bird flock foraging, the social behavior, inspired, put forward with the simplified.

Biologists CargiReynolds put forward a very influential birds gathered model. In his birds simulation model, each individual compliance: to avoid collision with neighborhood individuals, matches the speed of the neighborhood individuals, trying to fly to perceive the flock center this three rules to form simple driving birds gathered the centralized control algorithm, in a series of simulation experiments highlights the very close to the reality the birds gathered phenomenon. The results showed that the hovering bird form outline clear group, and encounter obstacles when birds division and the convergence process again. Inspired by this, simplified particle swarm optimization algorithm is proposed.

\section{Introduction}

Step 1. Initialization the location of the initial search point V0i X0i and its speed is usually in the range of allowed random, each particle Pbest coordinates is set to its current location, and calculate the corresponding individual extreme value (that is, the individual fitness value of the extreme value point and the optimal particle and the neighborhood is the best in the field of particle individual extremum, record the best value of particle number, and will be Nbesti set to the current location of the best particle.

Step 2. Evaluate each particle. Calculate the fitness value of particles, if better than the particle current individual extremum, sets the Pbest to the particle's position, and update the individual extremum.If all particles in the neighborhood of the particles in the individual extremum best is better than the current Nbesti, sets the Nbesti to the particle's position, record the serial number of the particles, and update Nbesti function value.

Step 3. The renewal of the particle. Update each particle's speed and position.

Step 4. Check whether meet the requirements for the end.If the current number of iterations to achieve the pre-set number of maximum (or minimum error requirement), is to stop the iteration, output optimal solutions, otherwise go to step 2. 


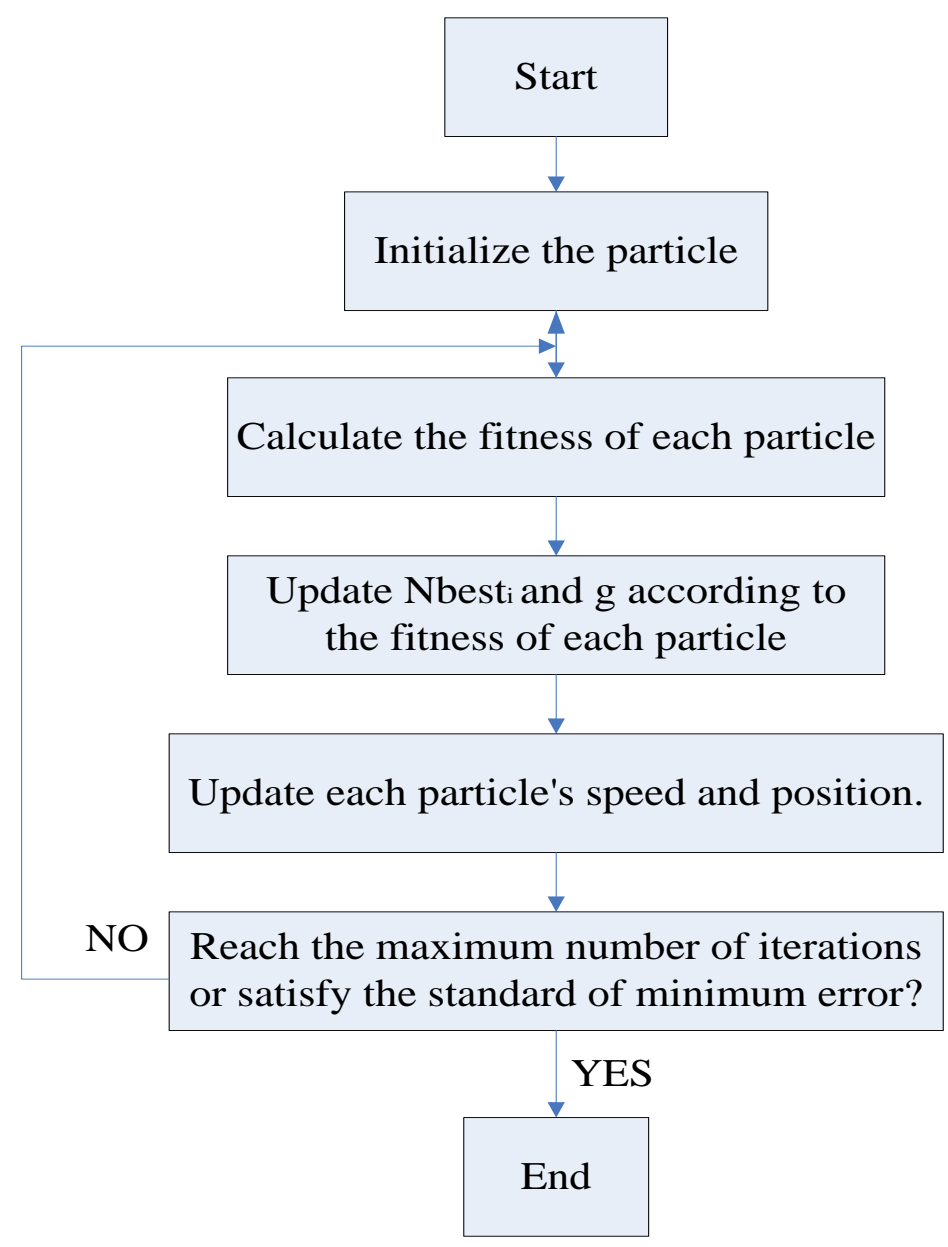

Fig. 1 The particle swarm algorithm to optimize algorithm flow chart

\section{The Simulation Results}

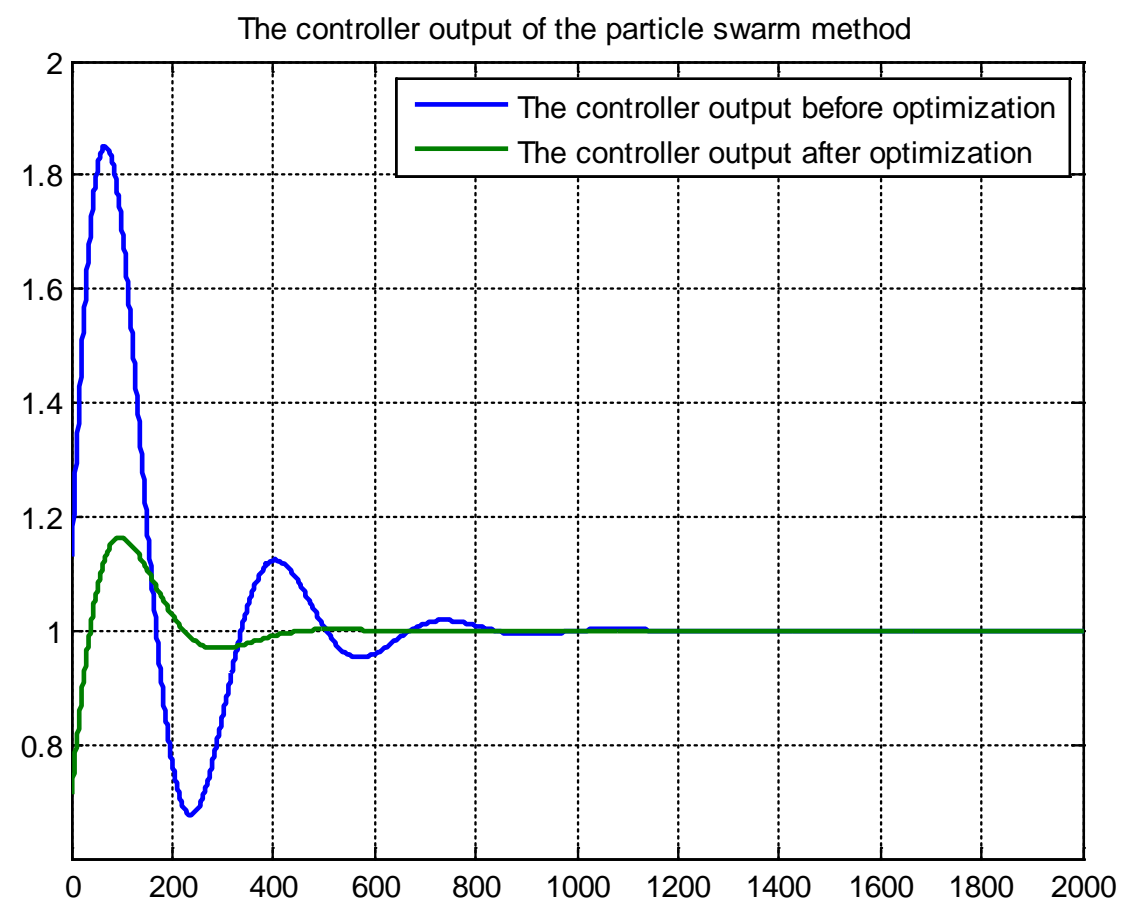

Fig. 2 The controller output of the particle swarm method 


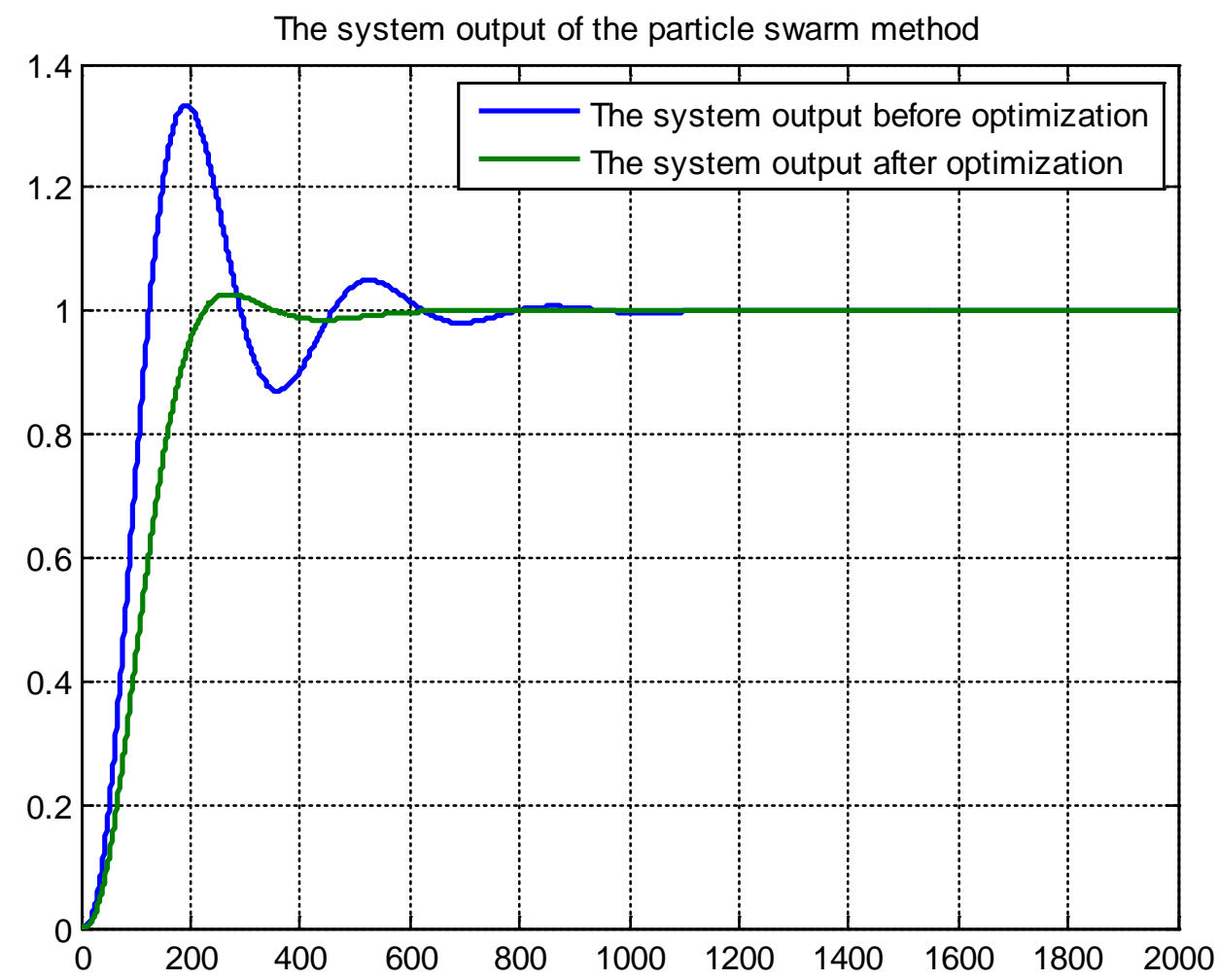

Fig. 2 The System output of the particle swarm method

Table 1 Three Scheme comparing

\begin{tabular}{ccc}
\hline parameters & Before optimization & after optimization \\
\hline Ti & 0.9000 & 1.4371 \\
The response curve of overshoot Mp & 60.0000 & 78.3192 \\
attenuation $\phi$ & $33.4492 \%$ & $2.7366 \%$ \\
Stable time ts & 0.8526 & 0.9936 \\
The steady-state value & 594 & 306 \\
Rise time tr & 1 & 225 \\
\hline
\end{tabular}

\section{Summary}

Calculated by the above after know the average relative error is 0.0159 , shows that the model has played a good effect, conform to the law of population development.

\section{References}

[1] Liu Jing. Particle swarm optimization (pso) algorithm and its parameter Settings, [J]. Sichuan academy of social sciences press, 2010, 2-3. 
[2] Zhen-su Hou Zhirong. Adaptive mutation particle swarm optimization algorithm [J]. Journal of electronics, 2004, 32 (3) : 416-416. The DOI: 10.3321 / j.i SSN: 0372-2112.2004.03.016.

[3] zhen-su Hou Zhirong. Adaptive mutation particle swarm optimization algorithm [J]. Journal of electronics, 2004, 32 (3) : 416-416. The DOI: 10.3321 / j.i SSN: 0372-2112.2004.03.016.

[4] NiQingJian, joey tang, han-cheng xing, etc. Based on the strategy of heterogeneous populations more dynamic probability of particle swarm optimization algorithm [J]. Journal of pattern recognition and artificial intelligence, 2014, (2) : 146-146. The DOI: 10.3969 / j.i SSN. 1003-6059.2014.02.008. 